\title{
Empiric Treatment for Suspected Malaria in the United States: A Case Report
}

\author{
Jonathan C. Li $^{1}$, Emma Lundsmith ${ }^{2}$ \\ 1. Internal Medicine-Pediatrics, ChristianaCare, Newark, USA 2. Internal Medicine, Thomas Jefferson University \\ Hospital, Philadelphia, USA
}

Corresponding author: Jonathan C. Li, lijc@upmc.edu

\begin{abstract}
Malaria in the United States is rare and most commonly presents among returning travelers from endemic areas. Diagnosis is classically dependent on a positive blood smear or polymerase chain reaction (PCR) test. The objective of this case report is to highlight a case of suspected malaria in a high-risk individual with negative diagnostic testing where a trial of empiric treatment was initiated based on clinical presentation after a thorough discussion of risks and benefits. However, empiric treatment based on a single case is limiting. We present a case of a 56-year-old man with extensive travel history throughout Asia, who presented after multiple episodes of unprovoked 24-hour fevers over the past seven years. A thorough rheumatologic and infectious inpatient workup was negative and oncology was consulted with low suspicion for malignancy. However, based on clinical presentation and history, malaria remained highly suspected and an empiric trial of anti-malarial treatment was initiated. One year after receiving treatment, the patient has not experienced any further febrile episodes. The efficacy of blood smears and PCR may be influenced by the malarial strain, as some species have low circulating biomass. Therefore, blood smears and PCR testing may not always be diagnostic. Clinical signs supportive of a malarial infection include fever, rigors, chills, hepato/splenomegaly, hyperbilirubinemia, and thrombocytopenia. Malaria is endemic to many regions outside of Africa, including Asia, and should be considered in any returning traveler with recurrent fevers.
\end{abstract}

Categories: Internal Medicine, Pathology, Infectious Disease

Keywords: malaria, diagnostic accuracy, travel medicine, vivax infections, undiagnosed fever

\section{Introduction}

Globally, malaria is a common cause of recurring fevers and is recognized to have high endemicity in subSaharan Africa and parts of Asia. However, malaria is also common outside of Africa and presents similarly but has distinct clinical differences. Blood smears with Giemsa stain are the gold standard for diagnosis [1]. However, some species of Plasmodium are less detectable in peripheral blood, and negative blood smears do not rule out the diagnosis $[2,3]$.

Received 10/28/2019 Review began 11/04/2019 Review ended 04/21/2020 Published 04/29/2020

() Copyright 2020 Li et al. This is an open access article distributed under the terms of the Creative Commons Attribution License CC-BY 4.0., which permits unrestricted use, distribution, and reproduction in any medium, provided the original author and source are credited.

\section{Case Presentation}

A 56-year-old man presented to the emergency department (ED) after experiencing a fever to $101^{\circ} \mathrm{F}\left(38.3^{\circ} \mathrm{C}\right)$ at home. His past medical history included intermittent febrile episodes over the past seven years and hypertension. His only medication was hydrochlorothiazide.

He experienced recurrent unprovoked febrile episodes (occurring every two to three months) for the past seven years. These episodes were acute in onset, without preference for day or night, and followed a stereotypic pattern. They began with chills/rigors followed by high-grade fever, severe day/nighttime diaphoresis, nausea, and sometimes palpitations. Spontaneous resolution typically occurred within 24-48 hours and left him feeling severely fatigued the following day. The highest fever he recorded during an episode was $104.1^{\circ} \mathrm{F}\left(40.1^{\circ} \mathrm{C}\right)$. He denied vomiting, diarrhea, abdominal pain, dysuria, arthralgias, myalgias, or unexpected weight loss in association with these episodes. Between episodes, he felt completely asymptomatic.

Five years prior to presentation, the patient resided in China for seven years. He also endorsed travel history to Taiwan, Malaysia, Thailand, Philippines, Korea, Japan, Costa Rica, and the Dominican Republic. He reports taking appropriate preventive measures against mosquitoes such as barriers and chemical prophylaxis while traveling. His first febrile episode occurred in Taiwan within the seven-year period when he was residing in China. Initially, after returning from China, he experienced dyspnea and chest pain with a negative cardiac and pulmonary workup.

He did not endorse any illicit drug use, tobacco use, and seldomly consumed alcohol. He was up to date with his preventive health screening exams. Family history was significant for rheumatoid arthritis in his sister. There was no other history of autoimmune disease or familial fever syndromes in his family.

The patient was admitted for systemic inflammatory response syndrome (SIRS) and for a diagnostic assessment for fever of unknown origin. Diagnostic labs and imaging are summarized in Table 1 and Table 2, respectively. Many of these were repeated during periods of fever without a significant difference in results. An extensive rheumatologic and infectious workup was negative and oncology was consulted with 


\section{Cureus}

low suspicion for malignancy. The infectious disease service was consulted and reported concern for malaria (particularly Plasmodium vivax (P. vivax) and Plasmodium ovale (P. ovale)) and brucellosis due to his travel history, although there lacked typical periodicity. They noted that splenomegaly, anemia,

thrombocytopenia, and mild transaminitis seemed consistent with malaria. A malaria expert was consulted at the Centers for Disease Control and recommended empiric treatment for malaria based on the patient's history and clinical findings. Following a negative glucose-6-phosphate dehydrogenase (G6PD) deficiency screen, the patient was started on empiric treatment with oral atovaquone/proguanil 1000/400 mg for three days and primaquine $30 \mathrm{mg}$ daily for 14 days.

\begin{tabular}{|c|c|c|}
\hline Lab & Measured value & Reference range \\
\hline High sensitivity troponin & $<6(\mathrm{~N})$ & < $19 \mathrm{ng} / \mathrm{L}$ \\
\hline D-dimer & $434(\mathrm{H})$ & $<204$ ng/mL \\
\hline International normalized ratio (INR) & $1.23(\mathrm{~N})$ & 0.8-1.19 \\
\hline Prothrombin time (PT) & $13.6(H)$ & $8.9-13.1 \mathrm{sec}$ \\
\hline Partial thromboplastin time (PTT) & $24(\mathrm{~N})$ & $24-35 \mathrm{sec}$ \\
\hline Urinalysis & Unremarkable & \\
\hline Urine drug screen & Negative & \\
\hline Urine culture & Negative & \\
\hline White blood cell & $7.6(\mathrm{~N})$ & 4.0-11.0 B/L \\
\hline Neutrophil & $91.40 \%$ & \\
\hline Lymphocytes & $5.00 \%$ & \\
\hline Monocytes & $2.90 \%$ & \\
\hline Eosinophils & $0.30 \%$ & \\
\hline Basophils & $0.10 \%$ & \\
\hline Red blood cell & $4.13(L)$ & 4.5-6 T/L \\
\hline Hemoglobin & $13.3(L)$ & $14-17 \mathrm{~g} / \mathrm{dL}$ \\
\hline Platelet & 115 (L) & $140-400$ B/L \\
\hline Blood cultures & Negative & \\
\hline Blood smear & Unremarkable & \\
\hline Lactate & $2.6(H)$ & $0.5-2.0 \mathrm{mmol} / \mathrm{L}$ \\
\hline C-reactive protein & $0.30(\mathrm{~N})$ & $\leq 0.80 \mathrm{mg} / \mathrm{dL}$ \\
\hline Erythrocyte sedimentation rate & $3(\mathrm{~N})$ & $0-20$ \\
\hline Thyroid stimulating hormone & $1.31(\mathrm{~N})$ & $0.3-5.00 \mathrm{ulU} / \mathrm{mL}$ \\
\hline Lactate dehydrogenase & $162(H)$ & 125-240 IU/L \\
\hline Haptoglobin & $75(\mathrm{~N})$ & $16-200$ mg/dL \\
\hline Total bilirubin & $1.3(\mathrm{H})$ & $1-1.2 \mathrm{mg} / \mathrm{dL}$ \\
\hline Aspartate aminotransferase & $45(\mathrm{H})$ & $0-40 \mathrm{IU} / \mathrm{L}$ \\
\hline Alanine aminotransferase & $76(\mathrm{H})$ & 0-44 IU/L \\
\hline Alkaline phosphatase & $56(\mathrm{~N})$ & 39-117 IU/L \\
\hline Iron & 28 (L) & $55-160 \mathrm{mcg} / \mathrm{dL}$ \\
\hline Total iron binding capacity & 247 (L) & $250-400 \mathrm{mcg} / \mathrm{dL}$ \\
\hline Iron saturation & $11 \%(L)$ & $20-55 \%$ \\
\hline Ferritin & $839(\mathrm{H})$ & $30-40 \mathrm{ng} / \mathrm{mL}$ \\
\hline Anti Smith Antibody & 8 & $0-89 \mathrm{U} / \mathrm{ml}$ \\
\hline Anti SS-A Antibody & 19 & $0-91 \mathrm{U} / \mathrm{ml}$ \\
\hline
\end{tabular}




\section{Cureus}

\begin{tabular}{|c|c|c|}
\hline Anti SS-B Antibody & 1 & $0-73 \mathrm{U} / \mathrm{ml}$ \\
\hline ANA screen & Negative & \\
\hline Anti dsDNA Antibody & 5 & $0-40 \mathrm{IU}$ \\
\hline Rheumatoid Factors & 11 & $<14 \mathrm{IU} / \mathrm{mL}$ \\
\hline A. phagocytoph. IgG & $<1: 64$ & $<1: 64$ \\
\hline A. phagocytoph. IgM & $<1: 20$ & $<1: 20$ \\
\hline Ehrlichia chaffeensis IgG & $<1: 64$ & $<1: 64$ \\
\hline Malaria Antigen Screen & Presumptive Negative & \\
\hline Lyme Ab screen & 1.75 (equivocal) & $<0.90$ Index \\
\hline Lyme Disease Ab (IgM) & Negative & \\
\hline Lyme Disease Ab (IgG) & Negative & \\
\hline Syphilis Antibody & Negative & \\
\hline Quantiferon Result & Negative & \\
\hline Tuberculosis Antigen & $\leq 0.000$ & \\
\hline Hep B Surf Antigen & Non-reactive & \\
\hline Hep C Antibody & Non-reactive & \\
\hline HIV Ab/Ag Result & Non-reactive & \\
\hline Cytomegalovirus polymerase chain reaction (PCR), Plasma & $<100 \mathrm{IU} / \mathrm{mL}$ & $<100 \mathrm{IU} / \mathrm{mL}$ \\
\hline Ebstein-Barr virus quantitative PCR, Plasma & Non detected & \\
\hline Mono Screen & Non-reactive & \\
\hline Babesia microti DNA reverse transcriptase-PCR & Not detected & \\
\hline Babesia microti DNA PCR & Not detected & \\
\hline
\end{tabular}

\section{TABLE 1: Blood tests}

Rheumatologic workup was negative for anti-Smith antibody, anti-SS-A antibody, anti-SS-B antibody, antinuclear antibodies (ANA), anti-dsDNA antibody, and rheumatoid factor. Infectious workup was negative for anaplasmosis, babesiosis, erlichiosis, malaria, Lyme, syphilis, tuberculosis, hepatitis B, hepatitis C, HIV, cytomegalovirus (CMV), and Epstein-Barr virus (EBV).

\begin{tabular}{|l|l|}
\hline \hline Imaging & Findings \\
\hline Chest X-ray & Unremarkable \\
\begin{tabular}{|l} 
Computed tomography (CT) angiography of \\
pulmonary embolism (PE)
\end{tabular} & $\begin{array}{l}\text { Negative for PE, mild interlobular interstitial thickening possibly representing pulmonary edema. } \\
\text { Cardiac morphology and pericardium were unremarkable. }\end{array}$ \\
\hline CT abdomen pelvis & $\begin{array}{l}\text { Mild splenomegaly measuring } 16 \times 12 \mathrm{~cm} \text {, trace amounts of free fluid in the lower pelvis of } \\
\text { uncertain etiology, and an enlarged lymph node near the liver. }\end{array}$ \\
\hline TABLE 2: Imaging findings & \\
\hline
\end{tabular}

Three weeks following discharge, the patient was seen for outpatient follow-up. The anemia was resolving with a hemoglobin of $13.3 \mathrm{~g} / \mathrm{dL}$; thrombocytopenia fully resolved with a platelet count of $169 \mathrm{~B} / \mathrm{L}$. Mild transaminitis persisted (aspartate aminotransferase (AST) 42 IU/L, alanine aminotransferase (ALT) 64 IU/L), total bilirubin had returned to normal $(0.7 \mathrm{mg} / \mathrm{dL})$, and alkaline phosphatase

(ALP) remained normal at $58 \mathrm{IU} / \mathrm{L}$. Though the improvement in labs may have simply reflected the resolution of SIRS, the patient reported no additional fevers during a telehealth follow-up one year later.

\section{Discussion}

The most common causes of recurrent febrile episodes include infectious, rheumatologic, and oncologic etiologies. Oncology did not suspect malignancy as the febrile episodes were too far inconsistent, complete 
blood cell count $(\mathrm{CBC})$ and smear were normal, and the patient was up to date on cancer screening without a high-risk family history. Rheumatology had low suspicion for an autoinflammatory disorder as the patient had a lack of arthralgias and rash, was completely asymptomatic between episodes, and workup for common autoimmune disorders were negative. Finally, workup for common infectious etiologies including viral, bacterial, and parasitic were negative. Despite this, based on the patient's travel history and clinical presentation, malaria was still highly suspected [4].

There are five Plasmodium species known to infect humans: falciparum, malariae, ovale, vivax, and knowlesi [1]. Plasmodium falciparum (P. falciparum) is the most common, accounting for $99 \%$ of known malaria cases, and generally is geographically-limited to sub-Saharan Africa [2,5].P. vivax is the most common species outside of Africa and accounts for $50 \%$ of non-African cases in the Americas, Eastern Mediterranean region, and Asia [2,5]. In Asia, the burden of $P$. vivax has been reported to be as high as $80 \%$ of the total global burden [4]. Other species in this region include Plasmodium knowlesi, P. ovale, and Plasmodium malariae [2,6].

Due to the infrequency of febrile episodes in this case, a Plasmodium species capable of producing hypnozoites seems most likely. Only P. vivax is confirmed to have a latent state in the liver (hypnozoites) with the ability to remain dormant for up to two years after primary infection [3]. P. ovale is suspected to have a hypnozoite stage as well, however, the evidence for this is limited and controversial [6]. Both $P$. vivax and $P$. ovale have reported relapse periods consistent with our patient's presentation, however, $P$. ovale less commonly produces clinical malaria and is more common in Africa than Asia [6,7].P. vivax is not commonly seen in Africa due to the lack of the Duffy antigen on erythrocytes in the region [2].

The classic episode of $P$. vivax infection begins with a prodrome of headache, anorexia, malaise, myalgias, and gastrointestinal symptoms for one or more days followed by a remitting fever [2]. Subsequent paroxysmal episodes occur in response to the rupturing of schizont-infected red blood cells (RBCs) [2]. These episodes begin with a stage of chills and rigors that last for approximately one hour, followed by fevers peaking 1-3 hours after rigors subside, and defervescence accompanied by diaphoresis and fatigue [2]. In comparison to $P$. falciparum, $P$. vivax induces an inflammatory response at a significantly lower parasite load [2]. Additionally, $P$. vivax has a predilection for reticulocytes, ultimately infecting $<2 \%$ of circulating erythrocytes while maintaining a greater capacity of causing severe anemia than $P$. falciparum [2]. The frequency of relapse depends on the infecting geographic strain of $P$. vivax [2]. Tropical strains relapse more frequently, from 1-6 months, and temperate strains relapse at intervals of eight months or greater [2].

\section{Clinical signs and abnormalities}

D-dimer is often elevated during malarial infections [8]. P. falciparum is well known to cause adherence of infected RBCs to the endothelium, causing damage and activation, and subsequently elevating D-dimer levels [8]. P. vivax is reported to have the same effect albeit with a 10 -fold lower capacity. D-dimer levels inturn reflect these pathophysiological differences [8].

In our case, our patient had a mild transaminitis and mild hyperbilirubinemia. Severe malarial infections can cause "malarial hepatopathy" which are defined by severe elevations of liver function tests [9]. These serologic elevations are higher in P. falciparum than $P$. vivax infections, suggesting that the etiology is more likely related to falciparum-specific etiologies (e.g. increased RBC cytoadherence) and not the presence of hepatic hypnozoites in P. vivax $[9,10]$. Our patient did not meet the criteria for malarial hepatopathy. We observed mild splenomegaly in our patient, a highly specific finding of malarial infection, and may simply reflect normal splenic filtration of abnormal RBCs, vascular congestion due to malaria, and organ-specific immune response against malaria $[10,11]$.

On CT, our patient was found to have mild pulmonary edema and free peritoneal fluid. These findings are rarely reported in non-severe malarial infections and consistent with the effects that malaria exerts on endothelium and microvascular function $[8,12]$.

Thrombocytopenia commonly occurs in malaria as well. The exact mechanism is not completely understood but centers around immunologic mechanisms damaging thrombocytes and causing excess platelet removal [13]. Thrombocytopenia is reported to have high sensitivity (94\%), high specificity (73\%), and a high negative predictive value (97\%) for malaria [14].

\section{Diagnostic studies}

Analysis of thick smear blood samples under light microscopy has greater sensitivity for diagnosing $P$. falciparum than $P$. vivax due to $P$. vivax having a preference for infecting pre-circulating reticulocytes $[3,15]$. Serologic testing for lactate dehydrogenase (LDH) and aldolase is useful but requires a moderately elevated parasitemia [3]. Antigen-detecting rapid diagnostic tests (RDTs) are now one of the most prevalent diagnostic tools used worldwide reaching areas where microscopy and polymerase chain reaction (PCR) are limited [16]. Overall, PCR testing tends to be the most sensitive as it is able to assess the presence of malaria, even with low parasite counts [17]. We identified only one other report of malaria with negative PCR [18]. In settings where laboratory testing is limited or high suspicion is not corroborated by diagnostic serologies (as in our case), other clinical findings have consistently been shown to support a diagnosis of malaria. Positive predictors include fever, non-pulsatile headache, chills and rigor, and periodicity to these symptoms [19,20]. Negative predictors include conjunctival suffusion, rash, respiratory rate $>28$, severe arthralgia/myalgia, throat congestion, fever $>40 \mathrm{C}^{\circ}$, and continuous fever $[19,20]$. A previous study reports that among travelers returning to the U.S, the most supportive clinical findings for malaria were splenomegaly, hepatomegaly, 
hyperbilirubinemia, and thrombocytopenia [10]. From this case, additional predictors may include imaging findings of non-specific fluid shifts (pulmonary edema, peritoneal fluid) reflecting malaria-induced endothelial dysfunction and hepatic lymphadenopathy reflecting hepatic hypnozoite activity.

When the malaria species is unknown, the World Health Organization recommends treatment under the assumed diagnosis of uncomplicated malaria by P. falciparum. This means that artemisinin-based combination therapies should be primarily utilized, mainly for its ability to overcomes chloroquineresistance. If the patient has G6PD, treatment should be centered around primaquine. Additional treatment with primaquine for 14 days in all transmission settings should be performed to prevent relapse [1].

\section{Conclusions}

Malaria is common and endemic to many countries outside of Africa (e.g. Asia), with P. vivax as the predominating, causative species. PCR is the best test when suspicious for $P$. vivax, however, it is not always diagnostic. Recurrence of febrile episodes maybe months to years apart. Clinical signs supportive of a malarial infection are fever, rigors, chills, hepato/splenomegaly, hyperbilirubinemia, and thrombocytopenia. Severe manifestations of malaria are life-threatening. G6PD should be tested for prior to empiric treatment. When the malarial organism is unknown, treatment is approached as if the patient has an uncomplicated $P$. falciparum infection.

\section{Additional Information \\ Disclosures}

Human subjects: Consent was obtained by all participants in this study. Jefferson - Office of Human Research issued approval N/A. OHR-34: Item 9: Case reports of one or two cases. (Three or more case reports in one series require IRB review). While one or two case reports do not require IRB review, they do require review by the Privacy Office. Conflicts of interest: In compliance with the ICMJE uniform disclosure form, all authors declare the following: Payment/services info: All authors have declared that no financial support was received from any organization for the submitted work. Financial relationships: All authors have declared that they have no financial relationships at present or within the previous three years with any organizations that might have an interest in the submitted work. Other relationships: All authors have declared that there are no other relationships or activities that could appear to have influenced the submitted work.

\section{Acknowledgements}

Special thanks to Dr. Lily Ackermann and Dr. Sean Clark-Garvey for their clinical preceptorship and Jennifer Wilson for her thoughtful and detailed revisions. Publication made possible in part by support from the Thomas Jefferson University + Philadelphia University Open Access Fund.

\section{References}

1. World Health Organization: guidelines for the treatment of malaria . (2015). Accessed: April 21, 2019: https://apps.who.int/iris/bitstream/handle/10665/162441/9789241549127_eng.pdf;jsessionid=009E8121020BEEEC28E783F8F4

2. Anstey NM, Douglas NM, Poespoprodjo JR, Price RN: Plasmodium vivax: clinical spectrum, risk factors and pathogenesis. Adv Parasitol. 2012, 80:151-201. 10.1016/B978-0-12-397900-1.00003-7

3. Dayananda KK, Achur RN, Gowda DC: Epidemiology, drug resistance, and pathophysiology of Plasmodium vivax malaria. J Vector Borne Dis. 2018, 55:1-8. 10.4103/0972-9062.234620

4. Kallinich T, Gattorno M, Grattan CE, et al.: Unexplained recurrent fever: when is autoinflammation the explanation?. Allergy. 2013, 68:285-296. https://doi.org/10.1111/all.12084

5. World Health Organization: world malaria report 2017. (2017). Accessed: April 21, 2019: https://apps.who.int/iris/bitstream/10665/259492/1/9789241565523-eng.pdf.

6. Groger M, Fischer HS, Veletzky L, Lalremruata A, Ramharter M: A systematic review of the clinical presentation, treatment and relapse characteristics of human Plasmodium ovale malaria. Malar J. 2017, 16:112. 10.1186/s12936-017-1759-2

7. Mueller I, Zimmerman PA, Reeder JC: Plasmodium malariae and Plasmodium ovale-the "bashful" malaria parasites. Trends Parasitol. 2007, 23:278-283. 10.1016/j.pt.2007.04.009

8. Meltzer E, Keller S, Shmuel S, Schwartz E: D-dimer levels in non-immune travelers with malaria . Travel Med Infect Dis. 2019, 27:104-106. 10.1016/j.tmaid.2018.05.004

9. Woodford J, Shanks GD, Griffin P, Chalon S, McCarthy JS: The dynamics of liver function test abnormalities after malaria infection: a retrospective observational study. Am J Trop Med Hyg. 2018, 98:1113-1119. 10.4269/ajtmh.17-0754

10. Taylor SM, Molyneux ME, Simel DL, Meshnick SR, Juliano JJ: Does this patient have malaria? JAMA. 2010, 304:2048-2056. 10.1001/jama.2010.1578

11. Buffet PA, Safeukui I, Deplaine G, et al.: The pathogenesis of Plasmodium falciparum malaria in humans: insights from splenic physiology. Blood. 2011, 117:381-392. 10.1182/blood-2010-04-202911

12. Barber BE, William T, Grigg MJ, et al.: Parasite biomass-related inflammation, endothelial activation, microvascular dysfunction and disease severity in vivax malaria. PLoS Pathog. 2015, 11:e1004558. 10.1371/journal.ppat.1004558

13. Naing C, Whittaker MA: Severe thrombocytopaenia in patients with vivax malaria compared to falciparum malaria: a systematic review and meta-analysis. Infect Dis Poverty. 2018, 7:10. 10.1186/s40249-018-0392-9

14. Arshad AR: Thrombocytopenia in malaria: can platelet counts differentiate malaria from other infections? . I Coll Physicians Surg Pak. 2015, 25:31-34. 01.2015/JCPSP.3134

15. Stauffer WM, Cartwright CP, Olson DA, et al.: Diagnostic performance of rapid diagnostic tests versus blood smears for malaria in US clinical practice. Clin Infect Dis. 2009, 49:908-913. https://doi.org/10.1086/605436 


\section{Cureus}

16. WHO, FIND, CDC (ed): Summary results of WHO product testing of malaria RDTs: round 1-8 (2008-2018) . 2018, 44. https://apps.who.int/iris/bitstream/handle/10665/276193/9789241514958-eng.pdf

17. Mfuh KO, Achonduh-Atijegbe OA, Bekindaka ON, et al.: A comparison of thick-film microscopy, rapid diagnostic test, and polymerase chain reaction for accurate diagnosis of Plasmodium falciparum malaria. Malar J. 2019, 18:73. https://doi.org/10.1186/s12936-019-2711-4

18. Bhome R, Bhome R: PCR negative cerebral malaria in a traveller returning from Mumbai . BMJ Case Rep. 2011, https://doi.org/10.1136/bcr.06.2011.4371

19. Shah V, Shah BK, Vadera B, Acharya HK: Clinical scoring system to predict malarial fever: a prospective study. Int J Med Public Health. 2011, 1:30-33. 10.5530/ijmedph.2.2011.7

20. Chandramohan D, Carneiro I, Kavishwar A, Brugha R, Desai V, Greenwood B: A clinical algorithm for the diagnosis of malaria: results of an evaluation in an area of low endemicity. Trop Med Int Health. 2001, 6:505-510. 10.1046/j.1365-3156.2001.00739.x 